\title{
SELEÇÃO QUE VESTE A CAMISA
}

\author{
Ricardo Dagnoni Huelsmanna, Lucas Ribeiro de Laiab e Eduardo Carasek, ${ }^{\mathrm{a}, *}$ \\ a Departamento de Química, Universidade Federal de Santa Catarina, 88040-900 Florianópolis - SC, Brasil \\ bepartamento de Química, Universidade do Estado de Santa Catarina, 89219-710 Joinville - SC, Brasil
}

Recebido em 29/04/2020; aceito em 10/02/2021; publicado na web em 26/02/2021

TEAM THAT GIVES ITS BEST. Football is the most practiced sport in the world, and admittedly one of the biggest cultural manifestations in Brazil. Its size and importance impact many fields of science. In this work, a playful and interactive activity is proposed, which allows the so beloved national sport and Chemistry to be united in a fun and reflective way, through the selection of the dream team. Each team can be formed by the desired tactical lineup, placing the player of your choice in the eleven available positions, describing its characteristic that makes it there. Even though it is derived from a competitive sport, there is no singular winner in this strategy, since everyone involved wins, due to the reflections and considerations developed, to the stimulus of creativity and to the fun involved in recreational activities like this.

Keywords: History of Chemistry; soccer; football; chemist; scientist.

\section{INTRODUÇÃO}

O futebol é reconhecidamente uma das mais proeminentes manifestações culturais do Brasil. ${ }^{1}$ Sua grandeza se dá por muitos fatores sociais, havendo intensa discussão das relações midiáticas ${ }^{2}$ e econômicas. ${ }^{3}$

Apesar da existência de diversas outras hipóteses, reconhece-se que o futebol moderno surgiu na Inglaterra, chegando ao Brasil com Charles Miller em $1895 .{ }^{4}$ Sendo inicialmente praticado por jovens da elite, rapidamente se popularizou e se espalhou. Uma vez dada sua disseminação em todo o território brasileiro, o futebol se tornou fator de integração nacional. ${ }^{5}$

O futebol - esporte mais praticado e assistido no mundo - está amplamente ligado à ciência. Uma breve busca na base de dados Scopus (pesquisando pelos termos soccer OR football em títulos, resumo e palavras-chave) resulta em mais de 45 mil trabalhos publicados. ${ }^{6} \mathrm{O}$ mais citado desses, publicado na revista Nature em 1985, rendeu a seus autores o Prêmio Nobel de Química (1996), pela descoberta do fulereno (então chamado Buckminsterfullerene). ${ }^{7}$ Esse trabalho traz em sua primeira página uma bola de futebol, dada semelhança estrutural da descoberta de H. W. Kroto, R. F. Curl e R. E. Smalley (e de seus auxiliares J. R. Heath e S. C. O'Brien, que ficaram de fora da premiação).

Dada sua magnitude e importância, o futebol também influencia e é influenciado por outras importantes áreas da ciência, como Medicina, ${ }^{8}$ Sociologia, ${ }^{9}$ Computação ${ }^{10}$ e Psicologia. ${ }^{11}$

Neste trabalho é descrita e discutida uma atividade lúdica e interativa, que permite unir de maneira divertida e reflexiva o tão amado esporte nacional com a Química, através de uma escalação do time dos sonhos.

\section{DESCRIÇÃO DA ESTRATÉGIA}

Uma atividade comum para amantes do futebol é escalar sua seleção ideal: superando barreiras temporais, sociais e espaciais, coloca-se em cada posição do time de futebol um jogador, cujas características e habilidades o fazem especial. Anualmente, por exemplo, a UEFA (União das Federações Europeias de Futebol, do

*e-mail: eduardo.carasek@ufsc.br inglês Union of European Football Associations) anuncia sua seleção ideal, formada pelos melhores jogadores de suas competições. ${ }^{12}$ Sites de esportes nacionais ${ }^{13,14} \mathrm{e}$ internacionais ${ }^{15,16}$ frequentemente lançam atividades parecidas, permitindo que internautas sejam os técnicos e escalem sua formação ideal, sejam jogadores do futebol de cada país, escalação ideal do seu time do coração, ou em momentos temáticos como a Copa do Mundo, entre outros.

Podendo ser elaborada de forma individual, em duplas de colegas ou mesmo em grupo, a atividade transcende a sala de aula e as discussões padrão dos cientistas importantes: buscando abordar a história da ciência numa atividade lúdica e com abordagem reflexiva, a proposta é escalar a melhor seleção de químicos e cientistas, que merecem recordação e homenagens, pelo seus estudos e discussões que impactaram a Química e a Ciência como um todo.

Cada time pode ser formado pela escalação tática que se deseja, colocando nas onze posições disponíveis o jogador (que é ou foi na verdade, um químico ou cientista) à sua escolha, descrevendo a característica que o faz estar ali. Por fim, da mesma forma, define qual o nome da equipe que agrupa toda sua seleção. Essas escolhas são feitas usando analogias entre as posições de cada jogador num time de futebol, com características das pesquisas e descobertas feitas pelo cientista em questão. Para elucidar a estratégia e enaltecer o empenho e dedicação de dezenas de cientistas, convocamos, apresentamos e discutimos duas seleções repletas de nomes que marcaram a evolução da Química em todo o mundo. Nomeadamente, o confronto imaginário será entre Linus Pauling Futebol Clube e Esporte Clube Alfred Werner.

\section{ESCALAÇÃO DO LINUS PAULING FUTEBOL CLUBE}

O primeiro time homenageia um dos poucos indivíduos laureados duas vezes com Prêmio Nobel (Química em 1952 e Paz em 1964). Linus Pauling, estadunidense nascido em Portland, um dos pioneiros na utilização de mecânica quântica para entender e descrever a ligação química, também teve grande participação na sociedade, principalmente no movimento anti-nuclear. ${ }^{17}$ Seu time está escalado no esquema 4-1-2-1-2 (Figura 1).

No gol o capitão John Dalton (\#1), goleiro-artilheiro que atua bem nos dois lados do campo, assim como o fez estudando desde meteorologia à teoria atômica. ${ }^{18} \mathrm{O}$ atomismo proposto por Dalton 
no século XIX é a base do conceito moderno de átomo, ${ }^{19}$ e ele, a base do time.

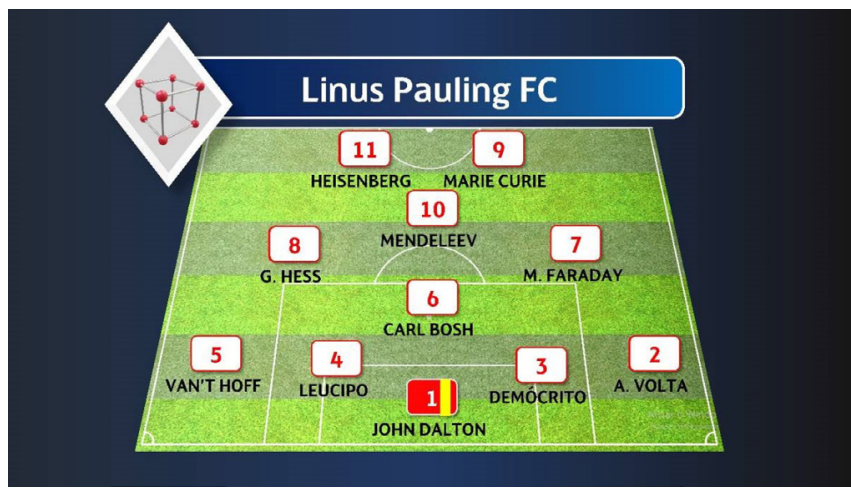

Figura 1. Escalação do time Linus Pauling FC

Alessandro Volta (Conte Alessandro Giuseppe Antonio Anastasio Volta, italiano da Lombardia) é o lateral direito (\#2): cheio de energia, é a pilha do time, dados seus estudos fundamentais da eletroquímica. Foi responsável pela invenção da pilha (bateria elétrica), fornecendo a primeira fonte de corrente contínua. ${ }^{20}$

Os zagueiros que sustentam o time representam a base do pensamento sobre a matéria: Demócrito (\#3) e Leucipo (\#4), filósofos gregos. Ambos são figuras centrais no desenvolvimento do atomismo filosófico e da teoria atômica do universo..$^{21-24}$

Na lateral esquerda está Jacobus Henricus Van’t Hoff (\#6), holandês ganhador do primeiro Prêmio Nobel de Química em 1901, ${ }^{25}$ que dá mais movimento ao time, ágil por seus estudos sobre dinâmica química. Van't Hoff foi muito abrangente em seus estudos, cujas ideiais passaram pelo carbono tetraédrico, termodinâmica, teoria das soluções, cinética química e petrologia, sendo assim um dos mais importantes cientistas da sua época. ${ }^{26}$

O volante escolhido é Carl Bosch (\#5), pois essa posição precisa de alguém saiba usar bem as condições de alta pressão. Tendo iniciado seus estudos em metalurgia e engenharia mecânica (de 1894 a 1896), o químico alemão começou a ler Química somente em 1896 (na Universidade de Leipzig). Recebeu com Friedrich Bergius o Prêmio Nobel de Química de 1931, pelo desenvolvimento de métodos químicos de alta pressão. ${ }^{27,28}$

No meio campo tem-se Michael Faraday (\#7) e Germain Hess (\#8). O camisa sete é constante (alusão a constante de Faraday, F, que representa a magnitude da carga elétrica por mol de elétrons). Faraday foi um dos maiores cientistas do século XIX. Sua maior contribuição foi no campo da eletricidade e do magnetismo, mas o químico e físico britânico contribuiu tanto no desenvolvimento quanto na divulgação da ciência. ${ }^{29}$ Ele foi pioneiro na produção de corrente elétrica a partir de um campo magnético, inventando também o primeiro motor e dínamo elétrico e demonstrando a relação entre a ligação química e a eletricidade. ${ }^{30}$

Já o número oito percorre diferentes caminhos, mas sempre com a mesma energia para encontrar o gol. Sua escolha para o time faz alusão à Lei de Hess, que afirma que a quantidade de calor envolvida numa reação química é a mesma, não importa quais e quantas etapas sejam envolvidas para obtenção do produto desejado. Essa lei desenvolvida pelo químico suíço-russo, preparou caminho para o desenvolvimento da termodinâmica química no final do século XIX, pelo famoso físico estadunidense Josiah Gibbs. ${ }^{31}$

$\mathrm{O}$ ataque é composto pela mulher que muito driblou o preconceito: Marie Curie ostenta a camisa 9, muito importante para o time. Nascida em Varsóvia (atualmente na Polônia) com o nome Marie Skłodowska, Curie ganhou duas vezes o Prêmio Nobel (Física em 1903 e Química em 1911) pelos estudos sobre radioatividade e descoberta dos elementos rádio e polônio. ${ }^{32}$

O cérebro do time, camisa 10, é Dmitri Mendeleev, aquele que sabe como organizar a periodicidade do time. Embora muitos outros cientistas se debruçaram anos em busca de organizar periodicamente os elementos conhecidos, Mendeleev (cientista russo) tem muito mérito em ser reconhecido como o pai da tabela periódica moderna. Dispondo os elementos conhecidos em ordem crescente de peso atômico, Mendeleev previu que alguns espaços vacantes seriam preenchidos por elementos ainda não conhecidos, tendo ele já previsto algumas de suas propriedades. ${ }^{33-35}$

Por fim, o número 11 é Werner Heisenberg, um finalizador que faz o goleiro sempre estar incerto, nunca sabendo a posição e a velocidade dos seus chutes. Físico e filósofo alemão, ganhador do Prêmio Nobel de Física em 1932 pela criação da mecânica quântica, ${ }^{36}$ Heisenberg foi responsável também pela formulação do princípio da incerteza, que estabelece que a posição e a velocidade de um objeto não podem ser medidas exatamente, ao mesmo tempo, nem mesmo em teoria. Ele também contribuiu na área de hidrodinâmica, estudou também o núcleo atômico, ferromagnetismo, raios cósmicos e partículas subatômicas. ${ }^{37}$

\section{ESCALAÇÃO DO ESPORTE CLUBE ALFRED WERNER}

Com uma escalação 4-3-3, o EC Alfred Werner é um time complexo (Figura 2). Werner, químico suíço nascido em 1866, com sua audácia e vasto conhecimento, fundou bases conceituais da Química de Coordenação, o que lhe rendeu o Prêmio Nobel de Química em 1913. ${ }^{38-40}$

No gol está postado Svante Arrhenius (\#1), que sabe como o jogo está dissociado, conseguindo se diluir no gol como ninguém, além de prever a velocidade do jogo enquanto sabe sua temperatura. Nascido na Suécia em 1859, Svante August Arrhenius (vencedor do Prêmio Nobel de Química em 1903) foi físico e físico-químico, responsável por variados estudos, desde sua teoria da dissociação eletrolítica até seu modelo do efeito estufa. Segundo a teoria de Arrhenius (1887), certas substâncias (eletrólitos) se dissolvem em água formando solução que conduz eletricidade, pela dissociação dos eletrólitos em partículas eletricamente carregadas (íons), mesmo quando não há corrente fluindo através da solução. ${ }^{41,42}$ Arrhenius também dá nome a uma equação (Equação 1) amplamente utilizada em físico-química, na qual é possível expressar a dependência das constantes de velocidade das reações químicas com a temperatura (é importante ressaltar que a equação de Arrhenius foi formulada inicialmente por J. J. Hood, mas Arrhenius mostrou que a relação é aplicável a quase todos os tipos de reações). ${ }^{43}$

$$
\mathrm{k}=\mathrm{Ae}^{\frac{-\mathrm{Ea}}{\mathrm{RT}}}
$$

Ou utilizando o logaritmo natural,

$$
\ln (\mathrm{k})=\ln (\mathrm{A})-\frac{\mathrm{Ea}}{\mathrm{R}} \frac{1}{\mathrm{~T}}
$$

Em que $\mathrm{k}$ é a constante de velocidade, A é a constante préexponencial, Ea é energia de ativação, $\mathrm{R}$ é a constante dos gases e $\mathrm{T}$ a temperatura. Essa equação prevê por exemplo que um pequeno aumento na temperatura da reação acarreta um aumento acentuado na magnitude da constante da taxa de reação, e também, que uma baixa energia de ativação significa uma reação rápida e uma alta energia de ativação significa uma reação lenta. ${ }^{4}$

Nas laterais estão Johannes Brønsted (\#6) e Thomas Lowry (\#2), que desenvolveram simultaneamente, mesmo separados, uma ótima 


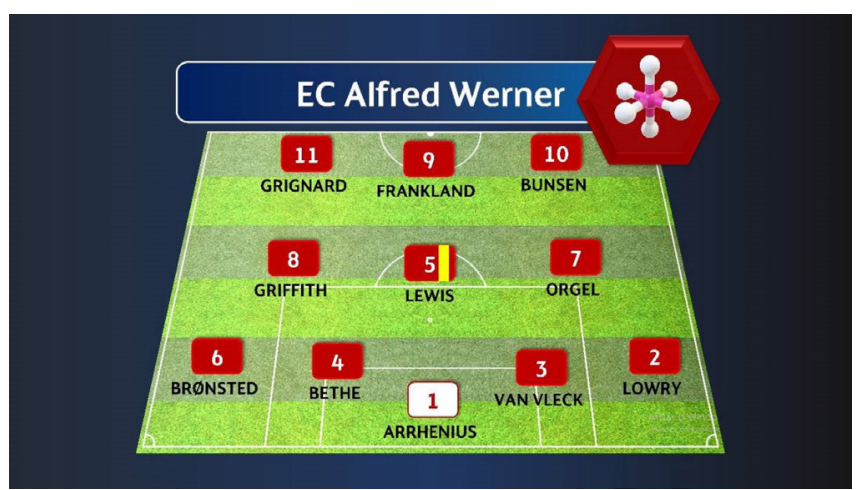

Figura 2. Escalação do time EC Alfred Werner

teoria no jogo proporcionando uma sinergia e dinâmica incrível entre eles.

Johannes Nicolaus Brønsted, nascido em 1879 na Dinamarca, ${ }^{45}$ e Thomas Martin Lowry (1874, Inglaterra), ${ }^{46}$ propuseram independentemente em 1923 a teoria ácido-base de doação de prótons (conhecida com teoria Brønsted-Lowry), na qual ácidos são quaisquer compostos que podem doar prótons, equanto bases são aqueles que são capazes de recebê-los. ${ }^{47}$ Essa teoria expandiu a compreensão dos ácidos e bases, superando a condição da teoria de Arrhenius que dependia do solvente ser água.

Os zagueiros são Van Vleck (\#3) e Hans Bethe (\#4), os melhores em estudar o campo: analisando a geometria do campo, conseguem ter um melhor posicionamento, conseguindo impedir até os ataques mais perigosos. Hans Albrecht Bethe foi um físico americano nascido em 1906, que auxiliou nos estudos da física quântica, aprimorando a compreensão dos processos atômicos responsáveis pelas propriedades da matéria e das forças que governam estruturas dos núcleos, ${ }^{48}$ enquanto John Hasbrouck Van Vleck (também norteamericano, nascido em 1899), contribuiu muito para a compreensão do comportamento dos elétrons em materiais sólidos magnéticos não cristalinos. ${ }^{49}$ Ambos ganharam o Prêmio Nobel de Física (1967 e 1977, para Bethe e van Vleck, respectivamente), mas são também bastante conhecidos nos estudos de Química Inorgânica pelas suas teorias do campo cristalino e do compo ligante, respectivamente.

O meio campo é governado por Gilbert Lewis (\#5), Leslie Orgel (\#7) e John Stanley Griffith (\#8).

O camisa cinco é o capitão do time, polivalente, consegue ter uma visão sobre quem deve receber ou passar a bola, auxiliando o time em campo, e assim sendo uma homenagem àquele tanto fez pela ciência e mesmo assim não ganhou o Prêmio Nobel. Gilbert Newton Lewis nasceu em 1875 em Massachusetts (EUA), e foi fundamental na teoria da ligação química, agrupando num único conceito todos os tipos de ligação. ${ }^{50}$ Além de muitos outros temas de estudo como termodinâmica e fosforescência, ${ }^{51}$ Lewis desenvolveu um complemento às teorias ácido-base, fugindo da obrigatoriedade de envolver prótons: numa reação química, um ácido de Lewis é uma espécie que recebe um par eletrônicos isolados, enquanto uma base de Lewis é a espécie que cede par de elétrons. ${ }^{52}$

Orgel e Griffith, por sua vez, são responsáveis pela transição, fazendo seu papel no campo ligante, permitindo assim que a bola flua livremente pelo time criando jogadas incríveis até o gol.

Leslie Eleazer Orgel foi um químico britânico nascido em 1927, que muito se destacou nos estudos da teoria do campo ligante, fornecendo ampla compreensão das propriedades estruturais e físicas dos complexos de metais de transição. Desenvolveu, por exemplo, os 'Diagramas de Orgel', diagramas de correlação que mostram as energias relativas dos termos eletrônicos em complexos de metais de transição. Orgel também foi muito importante em estudos da
Biologia Molecular, pioneiro no estudo das origens da vida. ${ }^{53}$ John Stanley Griffith, por sua vez, trabalhando com Orgel e deixou sua também sua marca na Química de Coordenação, especificamente na teoria do campo ligante. ${ }^{54}$

O camisa nove é Sir Edward Frankland, valente e organometálico, consegue juntar dois mundos diferentes, que até então eram opostos, armando jogada e atacando como ninguém. Nascido na Inglaterra em 1825, o químico Frankland foi um foi um dos primeiros investigadores no campo da química estrutural. Suas pesquisas no século XIX levaram a entender que átomos podem se combinar com número limitado de outros átomos (estabelecendo assim a teoria da valência, base da química estrutural moderna), tendo sido importante também no estudo dos compostos organometálicos..$^{55,56}$

Com a camisa 10 está Robert Bunsen, colocando fogo no jogo. Robert Wilhelm Bunsen foi um químico alemão nascido em 1811 e, junto de Gustav Kirchhof, observou que os elementos emitem luz em comprimentos de onda característicos. Tais observações abriram o campo de análise do espectro, o que foi de grande importância no estudo do Sol e das estrelas, fazendo com que Bunsen e Kirchhof descobrissem, por exemplo, os elementos césio e rubídio. Bunsen também inventou calorímetros e bomba de filtro, ficando mais conhecido pelo bico de Bunsen, o qual apenas desenvolveu de forma secundária, a partir de desenhos de Peter Desdega e Michael Faraday. ${ }^{57}$

E por último, porém sendo um exímio atacante, Victor Grignard, famoso por suas jogadas de contra-ataque "reação de Grignard", conseguindo quebrar qualquer defesa. François-Auguste-Victor Grignard foi um químico francês nascido em 1871 e agraciado com o Prêmio Nobel de Química de 1912 (junto de Paul Sabatier), pelo desenvolvimento da reação de Grignard. Seu trabalho com compostos de organomagnésio abriu uma ampla área de síntese orgânica. ${ }^{58}$

\section{CONCLUSÕES}

É importante ressaltar que essas escalações derivam de experiências e criatividade dos autores quando foi desenvolvido este trabalho. É sabido que muitos importantes cientistas não foram contemplados, mas que com certeza serão nas próximas seleções a serem escaladas por você, leitor. Em alguns momentos, as características mais conhecidas de cada cientista podem ser utilizadas, assim como detalhes mais profundos e específicos.

Uma dica é a criação de seleções temáticas, sejam pelas clássicas áreas da Química como um fantástico jogo entre Química Analítica x Química Inorgânica, ou mais especificamente em temas como Cromatografia x Espectrometria de Absorção Atômica.

Por meio dessa estratégia é possível trabalhar e discutir uma abordagem histórica e construtivista da Química. Por exemplo, com a elaboração de um "time dos sonhos" dos modelos atômicos, podese perceber que, com o surgimento de uma nova teoria, nem sempre anterior é invalidada, servindo muitas vezes como um complemento ou auxiliando a explicar determinado fenômeno na qual a outra falhava em explicar. Um outro exemplo seria sobre as teorias de ácidos e bases, nas quais conforme estas evoluíram, conseguiram explicar melhor como funcionava a reação em meio aquoso e com substâncias que não necessariamente liberam $\mathrm{H}^{+}$ou $\mathrm{OH}^{-}$.

O mais importante é que mesmo sendo derivado de um esporte competitivo, não há um vencedor singular. Todos os envolvidos saem ganhando, pelas reflexões e considerações desenvolvidas, pelo estímulo da criatividade e pela diversão envolvida em atividades lúdicas como esta.

\section{AGRADECIMENTOS}

Os autores agradecem à Universidade Federal de Santa Catarina 
e à Universidade do Estado de Santa Catarina. O presente trabalho foi realizado com apoio da Coordenação de Aperfeiçoamento de Nível Superior - Brasil (CAPES) - Código de Financiamento 001.

\section{REFERÊNCIAS}

1. Reis Filho, J.; Abib, P. R. J. Em Capacitação docente e responsabilidade social: aportes pluridisciplinares; Tenório, R. M., Silva, R. de S., orgs.; EDUFBA: Salvador, 2010.

2. Gastaldo, É.; Sociologias 2009, 353.

3. Benevides, B. Í. L.; Santos, S. M. dos; Cabral, A. C. de A.; Rev. econ. contemp. 2017, 21.

4. Máximo, J.; Estud. av. 1999, 13, 179.

5. Mascarenhas, G. Em Visões do Brasil: estudos culturais em Geografia; Barthe-Deloizy, F., Serpa, A., orgs.; EDUFBA: Salvador, 2012.

6. https://www.scopus.com/search/form.uri, acessada em fevereiro 2021.

7. Kroto, H. W.; Heath, J. R.; O’Brien, S. C.; Curl, R. F.; Smalley, R. E.; Nature 1985, 318, 162.

8. Impellizzeri, F. M.; Meyer, T.; Journal of Sports Sciences 2016, 34, 2175.

9. Cleland, J.; Cashmore, E.; International Review for the Sociology of Sport 2016, 51, 27.

10. Lee, J.-M.; Chun, H.-Y.; Dilmurod, Y.; Ko, K.-S.; Kang, T.-K. In Advances in Intelligent Systems and Computing; Kim, J.-H., Karray, F., Jo, J., Sincak, P., Myung, H., eds.; Springer International Publishing: Cham, 2017; Vol. 447, pp. 373.

11. Alesi, M.; Bianco, A.; Luppina, G.; Palma, A.; Pepi, A.; Perceptual and Motor Skills 2016, 122, 27.

12. https://www.uefa.com/insideuefa/mediaservices/mediareleases/ newsid=2637789.html, acessada em fevereiro 2021 .

13. https://globoesporte.globo.com, acessada em fevereiro 2021.

14. https://www.uol.com.br/esporte/, acessada em fevereiro 2021.

15. https://www.skysports.com/football/news/11661/11889057/pick-yourpremier-league-team-of-the-decade, acessada em fevereiro 2021

16. https://www.bbc.com/sport/football/50502851, acessada em fevereiro 2021.

17. https://www.nobelprize.org/prizes/chemistry/1954/pauling/facts/, acessada em fevereiro 2021.

18. https://www.britannica.com/biography/John-Dalton, acessada em fevereiro 2021.

19. https://www.sciencehistory.org/historical-profile/john-dalton, acessada em fevereiro 2021.

20. https://www.britannica.com/biography/Alessandro-Volta, acessada em fevereiro 2021.

21. https://plato.stanford.edu/entries/democritus/, acessada em fevereiro 2021.

22. https://www.britannica.com/biography/Democritus, acessada em fevereiro 2021.

23. https://www.britannica.com/biography/Leucippus, acessada em fevereiro 2021.

24. https://www.iep.utm.edu/leucippu/, acessada em fevereiro 2021.

25. https://www.nobelprize.org/prizes/chemistry/1901/hoff/biographical/, acessada em fevereiro 2021.

26. Chagas, A. P.; Quim. Nova Esc. 2001, 14, 25.

27. https://www.britannica.com/biography/Carl-Bosch, acessada em fevereiro 2021.
28. https://www.nobelprize.org/prizes/chemistry/1931/bosch/biographical/, acessada em fevereiro 2021.

29. Baldinato, J. O.; Porto, P. A.; Quim. Nova Esc. 2008, 30, 16.

30. https://www.britannica.com/biography/Michael-Faraday, acessada em fevereiro 2021.

31. https://www.britannica.com/biography/Germain-Henri-Hess, acessada em fevereiro 2021.

32. de Farias, R. F.; Quim. Nova Esc. 2001.

33. Leite, H. S. A.; Porto, P. A.; Quim. Nova 2015.

34. Lima, G.; Barbosa, L.; Filgueira, C.; Quim. Nova 2019

35. Bensaude-Vincent, B.; Dmitri Mendeleev, disponível em https://www. britannica.com/biography/Dmitri-Mendeleev, acessada em fevereiro 2021.

36. https://www.nobelprize.org/prizes/physics/1932/summary/, acessada em fevereiro 2021.

37. https://www.britannica.com/biography/Werner-Heisenberg, acessada em fevereiro 2021.

38. Santos, L. M.; Sarto, L. E.; Bozza, G. F.; de Almeida, E. T.; Rev. Virtual Quim. 2014, 6.

39. https://www.britannica.com/biography/Alfred-Werner, acessada em fevereiro 2021.

40. https://www.nobelprize.org/prizes/chemistry/1913/werner/biographical/, acessada em fevereiro 2021

41. https://www.britannica.com/biography/Svante-Arrhenius, acessada em fevereiro 2021.

42. https://www.nobelprize.org/prizes/chemistry/1903/arrhenius/ biographical/, acessada em fevereiro 2021 .

43. https://www.britannica.com/science/Arrhenius-equation, acessada em fevereiro 2021.

44. Levine, I. N.; Physical Chemistry, $6^{\text {th }}$ ed., McGraw-Hill: New York, 2009.

45. https://www.britannica.com/biography/Johannes-Nicolaus-Bronsted, acessada em fevereiro 2021

46. Jensen, W. B.; Educ. Quím. 2016, 27, 175.

47. https://www.britannica.com/science/Bronsted-Lowrytheory\#ref1279911, acessada em fevereiro 2021.

48. https://www.britannica.com/biography/Hans-Bethe, acessada em fevereiro 2021.

49. https://www.britannica.com/biography/John-Van-Vleck, acessada em fevereiro 2021.

50. Filgueiras, C. A. L.; Quim. Nova 2016.

51. https://www.britannica.com/biography/Gilbert-N-Lewis, acessada em fevereiro 2021.

52. Housecroft, C.; Sharpe, A. G. Inorganic Chemistry, $3^{\text {rd }}$ ed., Prentice Hall: Upper Saddle River, 2007.

53. Joyce, G. F.; Nature 2007, 450, 627.

54. Griffith, J. S.; Orgel, L. E.; Quarterly Review of the Chemical Society 1957, 11,381 .

55. Nogueira, H.; Porto, P.; Quim. Nova 2018.

56. https://www.britannica.com/biography/Edward-Frankland, acessada em fevereiro 2021.

57. https://www.britannica.com/biography/Robert-Bunsen, acessada em fevereiro 2021.

58. https://www.britannica.com/biography/Victor-Grignard, acessada em fevereiro 2021. 